\title{
Virtual screening of inhibitory agents against SARS-CoV2
}

\author{
Muniba Parvez ${ }^{1}$, Muhammad Bilal ${ }^{1}$, Robaica Khan', Maham Chaudhry¹, Sajid Asghar², \\ Sajid Khan Sadozai ${ }^{3}$, Ayesha Sajjad ${ }^{1}$, Zulekha Mughal', Kashif Iqbal ${ }^{1}$, Samar Akhtar ${ }^{4}$, \\ Fahad Hassan $\operatorname{Shah}^{5 *}$, Saad Salman ${ }^{1 *}$ \\ ${ }^{1}$ Department of Pharmacy, The University of Lahore, Islamabad Campus, Pakistan \\ ${ }^{2}$ Department of Pharmaceutics, Governemnt College University Faisalabad, Pakistan \\ ${ }^{3}$ Department of Pharmacy, Kohat University of Science and Technology, Kohat, Pakistan \\ ${ }^{4}$ Yusra Institute of Pharmaceutical Sciences, Islamabad, Pakistan \\ ${ }^{5}$ Centre of Biotechnology and Microbiology, University of Peshawar, Pakistan
}

\begin{abstract}
Severe Acute Respiratory Syndrome (SARS-CoV2) infected about 93 million people and killed over two million worldwide. The disease transmits very quickly, therefore; due to its severity and widespread the World Health Organization has declared this menace as 'Global Pandemic'. An urgent need was felt to manage this disease through aggressive and efficient research process all over the globe. That's why drug re-purposing of 212 chemical entities (CEs) against SARSCOV2 was found to be one of the efficient ways in finding new indications of already discovered drugs amisdst of the discovery of a new drug. Results of this study revealed that out of 212 CEs, only Etodolac forms a hydrogen $(\mathrm{H})$-bond with a relatively low energy and active central fragment, demonstrating more significant interaction with SARS-CoV2 viral proteins. Other CEs exhibit good pharmacokinetics properties with the least acute toxicity through ADMET analysis. We also discovered other therapeutic applications of these CEs through Molinspiration. Etodolac, a non-steroidal anti-inflammatory drug forms H-bonding with $5.6 \mathrm{kcal} / \mathrm{mol}$ binding energy with active residues of this receptor. This drug created H-bonding with PHE326 and PRO328, with pyridine group, and was found more suitable to control SARS-CoV2.
\end{abstract}




\section{Introduction}

Severe Acute Respiratory Syndrome (SARS-CoV2) is an acute virus reported in Wuhan, China [1] causing COVID-19. The virus was very lethal and its spread was so rapid, that only in one year the pandemic reached 219 countries around the globe. The World Health Organization (WHO) informed that till mid-January 2021, COVID-19 infected nearly 93 million people and the mortality rate reached over two million worldwide [2]. The disease was named COVID-19 on $19^{\text {th }}$ February 2020 and the virus was called SARS-CoV2 [1]. This lethal disease is transmitted at a rapid rate in other parts of the world including the United States of America, which very soon became the global epicenter of COVID-19 [3]. Information on the animal origin of the Coronavirus outbreak curve, incubation time, pathogenesis, age span, viral spectroscopy, and clinical responses to antiviral, etc was too limited and much difficult to study [3]. Recent studies exhibit that respiratory and digestive organs were mostly affected by SARS-CoV2. Among the four major coronavirus types, Gamma-coronavirus and Deltacoronavirus mainly infect birds, while Beta-coronavirus and Alpha-coronavirus mostly infect mammals [4]. Coronavirus infects people of all ages, however, people already suffering from asthma, heart disease, diabetes, and geriatric people were the main target of this virus [4]. Within 2-14 days of viral exposure symptoms of COVID-19 normally appear [3] and [5]. Research shows that SARS-CoV2 is found in $70 \%$ of alveolar macrophages that were located in the air sacs of the lungs. High expression of genes that were involved in inflammation was exhibited by the cells nursing the virus. The findings suggested that, once the virus reaches the lungs, it can infect macrophages, which respond by producing inflammatory molecules that attract $\mathrm{T}$ cells. $\mathrm{T}$ cells, in turn, produce a protein that stimulates macrophages to make more inflammatory molecules. This persistent lung inflammation could lead to some of the lifethreatening consequences of Coronavirus infection [6]. One of the most effective ways to treat the patients suffering from Coronavirus infection as suggested by the researchers was the use of NSAIDs. WHO, therefore, recommended that globally coordinated efforts were needed to quickly stop the further spread of this deadly virus [7]. 
Extensive research efforts had been made by scientists to investigate the process of discovering potent therapeutic agents and specific target sites by using different means like Computational Drug Screening and Artificial Intelligence [8]. Because de novo drug designing is very lengthy, time-consuming, and expensive, therefore, scientists focused on another fundamental and alternative approach that helps in finding new indications for already discovered drugs, also known as drug repurposing or drug re-profiling [9]. Computational Drug Re-profiling was an economical and less time-consuming approach in this venture. This approach is much beneficial when the selected drugs were already approved by Food and Drug Administration (FDA) and had well-defined pharmacodynamics, pharmacokinetics, adverse effects, tolerability, and safety profile. We also have data on their post-marketing surveillance [10]. This technique comprises of two approaches mainly. Firstly, a disease-based approach, in which disease characteristics were compared by using datasets to find a new indication for already existing drugs. Secondly, a target-based approach in which target-drug interactions were established by allowing drug and target to interact with each other. Protein Data Bank (PDB) provided about 110 protein structures that were linked with SARS-CoV2 for better insight of structural binding sites of virus that provide a basis for rational drug targeting [10]. From Virtual Screening the scientists had found some new anti-viral drugs through Molecular Docking [11]. It is an important approach in the repurposing of the drug which was performed by analyzing the targeted protein catalytic site and finding the drug molecules that bind with it. However, in the majority of human and viral targets; unfortunately, the three-dimensional structure of proteins was unavailable that limits traditional structure-based virtual screening methods. A high risk of viral resistance may also occur when targeting a single viral protein due to the viral genome's speedy evolution. The drugs that act upon multiple targets were considered to be more effective against Human Corona Virus as it makes resistance occurring problems much difficult. Multi-drug targeting is another approach in this venture as it hinders the virus from mutation by inhibiting its function during various stages of its life-cycle [12]. The primary Proteases (3CLpro) was the most significant protein and a key target in this aspect as it plays an important role in viral replication, thus halting this enzyme function will ultimately inhibit virus multiplication. Other proteins that we had previously studied included the proteins that were involved in host cell binding, host-specific enzymes, and virulence proteins. Furthermore, the speed of the viral infection was significantly reduced by blocking the viral cycle at any stage thus providing time to the immune system to combat the 
virus. Interlinking a particular viral cycle inhibiting application and a procedure related to ensemble clustering was used (in which errors were compensated through multiple algorithmic predictions) [13]. We had implemented a drug re-profiling scheme in which we detected computationally feasible binding sites for the viral proteins whose three-dimensional structures were experimentally determined. Then, we find the drug molecules that were supposed to interact with these binding sites and classify them according to the following criteria: number of targeted sites anticipated by the molecules and their affinity to bind these targets. This multitarget approach was previously utilized in our studies. The Drug Bank had explored a variety of compounds but the main focus of this study was on those compounds that were approved by the FDA and other world drug agencies and commercialized drugs which were economical and easily available so that they could be used immediately for the clinical trial; 4) Drugs that interacted with different proteins simultaneously were of our foremost interest. From the previous analysis, many molecules were eminent from the rest of the compounds. In the second analysis of virtual screening, we then compared those molecules which were presently used in COVID-19. This study expressed that the compounds of drugs discovered in the first analysis compare more suitably to the ones used in clinical trials. Herein, we report different chemical entities active against targeted proteins of SARS-CoV2.

\section{Materials and methods}

A total of 212 drugs (Anti-Inflammatory and Anti-Psychotic) approved by the FDA, were procured from PubChem and minimized using the PRODRG Server. From RCSB Protein Databank ID: 6M71 we downloaded RNA-dependent polymerase enzyme, a receptor of SARS$\mathrm{CoV} 2$, to test the interaction of these ligands. The 3-D structure of this receptor was prepared and minimized using ModRefiner and then the active site was predicted with Prank Web Server. PyRx 0.9 was used to screen these ligands for the determination of probable interaction against

this receptor. Through SwissADME and GUSAR databases, ligands that had proven successful in screening were further investigated for pharmacokinetics properties (absorption, metabolism distribution, excretion, and toxicity). 


\section{Results}

These 212 ligands were analyzed for drug-likeness and lead likeness criteria using SwissADME, to obtain efficacious compounds before screening analysis (Supplementary Table 1-2). From these, only 122 qualified these criteria and were further used for virtual screening against SARSCoV2 RNA Dependent RNA Polymerase (RdRP) enzyme. The results obtained from screening studies were further classified based on the type of chemical interaction of ligand and receptor, specifically, hydrogen interaction of ligands with the predicted active pocket of RdRP enzyme of SARS-CoV-2 were considered. Etodolac, an NSAIDs drug showed hydrogen bonding with 5.6 $\mathrm{kcal} / \mathrm{mol}$ of binding energy with the active residues of this receptor. This drug created hydrogen bonding with PHE326 and PRO328 with the pyridine group whereas the phenolic group formed pi-pi interaction with PHE396 respectively.

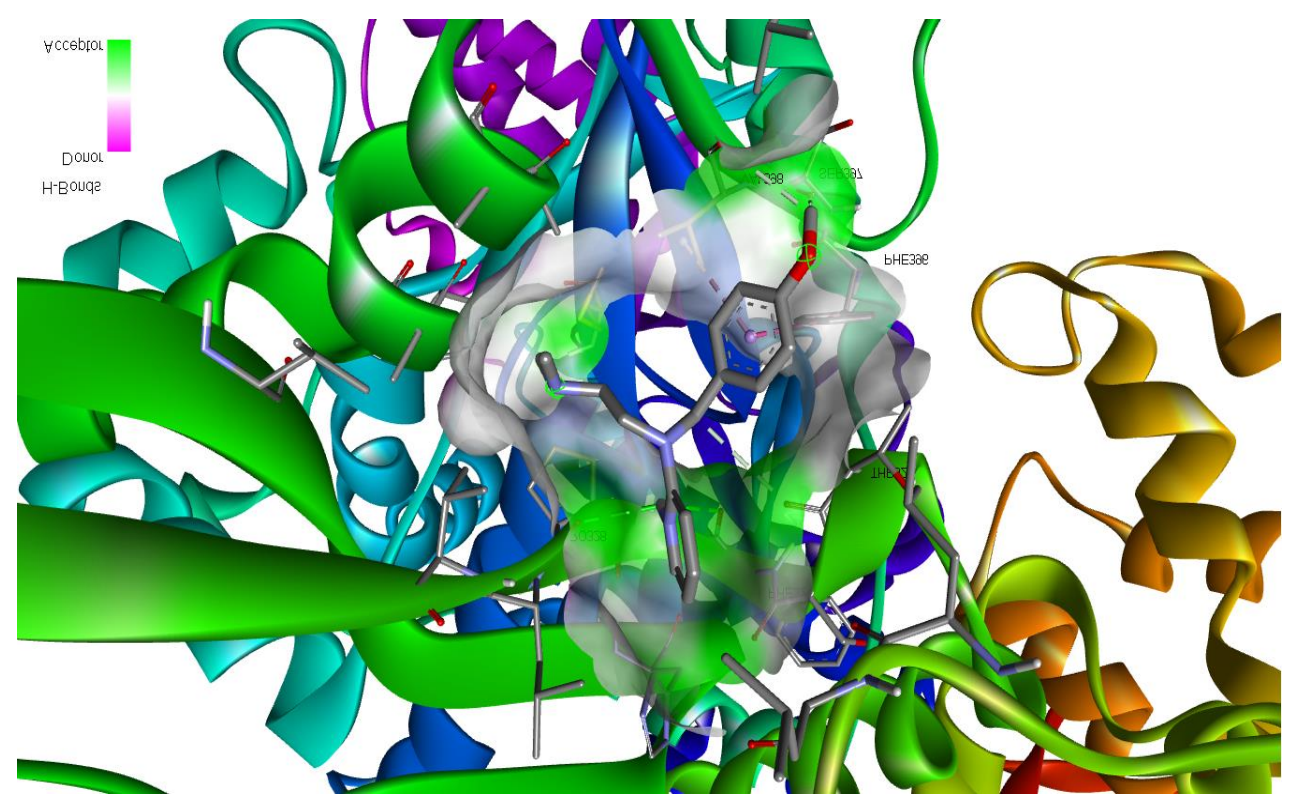

Fig 1. 3D Binding orientation of Etodolac with SARS-CoV2 RNA Dependent RNA Polymerase (RdRP) enzyme 


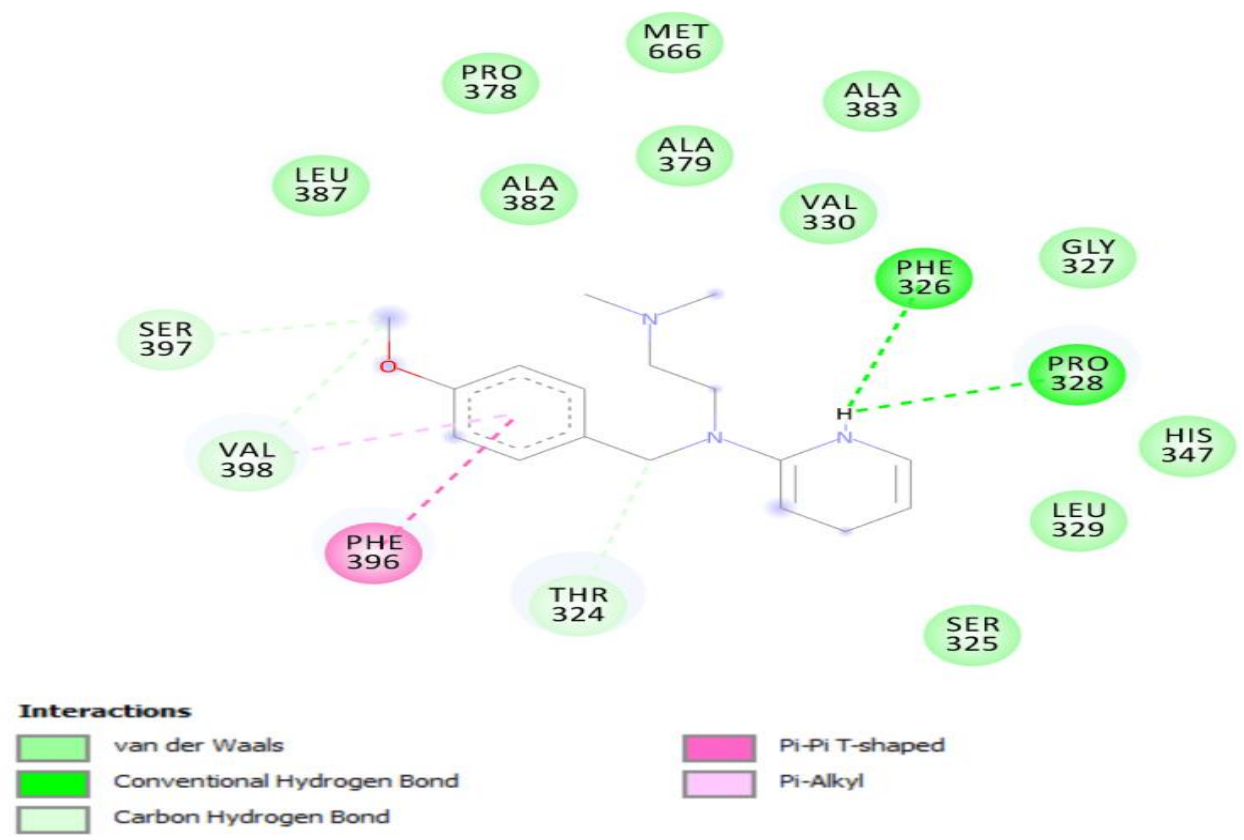

Fig 2. Participation of Active Amino Acids Residues of SARS-CoV2 (RdRP) enzyme with Etodolac

\section{Acute toxicity studies}

Acute toxicity study is based on examining the unfavorable side effects that a drug may cause to test organism after single or multiple exposures through various routes including oral, intraperitoneal, intravenous, or subcutaneous route. GUSAR was used to check the acute toxicity of desirable compounds (Table 1). GUSAR analyzes the compounds based on their chemical structures and compares the acquired data with SYMYX MDL toxicity and Organization of Economic Cooperation and Development (OECD) chemical classification database. The lethal dose of etodolac for intraperitoneal route is $-0,439 \log 10(\mathrm{mmol} / \mathrm{kg})$, for Intravenous route 0.690 , oral route 0.538 and 0.014 for subcuatenous route (Table 1). The obtained results from GUSAR chemically classified the screened drug in different classes through the OECD chemical classification system. 
Table 1. GUSAR toxicity study of Etodolac

\begin{tabular}{|c|c|c|c|c|c|c|c|c|}
\hline $\begin{array}{l}\text { DRUG } \\
\text { S }\end{array}$ & $\begin{array}{l}\text { Rat IP LD50 } \\
\text { Log10(mmol } \\
/ \mathrm{kg})\end{array}$ & $\begin{array}{l}\text { Rat IV } \\
\text { LD50 } \\
\log 10(\mathrm{mmol} \\
/ \mathrm{kg})\end{array}$ & $\begin{array}{l}\text { Rat Oral } \\
\text { LD50 } \\
\log 10(\text { mmol } \\
\text { /kg) }\end{array}$ & $\begin{array}{l}\text { Rat SC } \\
\text { LD50 } \\
\operatorname{log10}(\mathrm{mmol} \\
/ \mathrm{kg})\end{array}$ & $\begin{array}{l}\text { Rat IP } \\
\text { LD50 } \\
\text { Classificat } \\
\text { ion }\end{array}$ & $\begin{array}{l}\text { Rat IV } \\
\text { LD50 } \\
\text { Classificat } \\
\text { ion }\end{array}$ & $\begin{array}{l}\text { Rat Oral } \\
\text { LD50 } \\
\text { Classificat } \\
\text { ion }\end{array}$ & $\begin{array}{l}\text { Rat SC } \\
\text { LD50 } \\
\text { Classificat } \\
\text { ion }\end{array}$ \\
\hline $\begin{array}{l}\text { Etodol } \\
\text { ac }\end{array}$ & 0,348 & $-0,690$ & 0,538 & 0,014 & Class 5 & Class 4 & Class 4 & Class 4 \\
\hline
\end{tabular}

\section{Pharmacokinetic properties}

Before animal and clinical trials, it is a pre-requisite to find out the pharmacokinetic properties of the compounds, their effects on organisms concerning absorption, metabolism, distribution, and excretion. From the SwissADME data, we obtained information regarding the drug-likeness and pharmacokinetics properties of screened compounds. The water solubility and gastrointestinal absorption values of this compounds were high. The compounds can cross BBB (blood-brain barrier) and satisfy the criteria of drug-likeness with zero violations. Along with that their physicochemical properties and lipophilicity were also checked. The data was then compiled and given in Table 2. 
Table 2. SwissADME study of Etodolac.

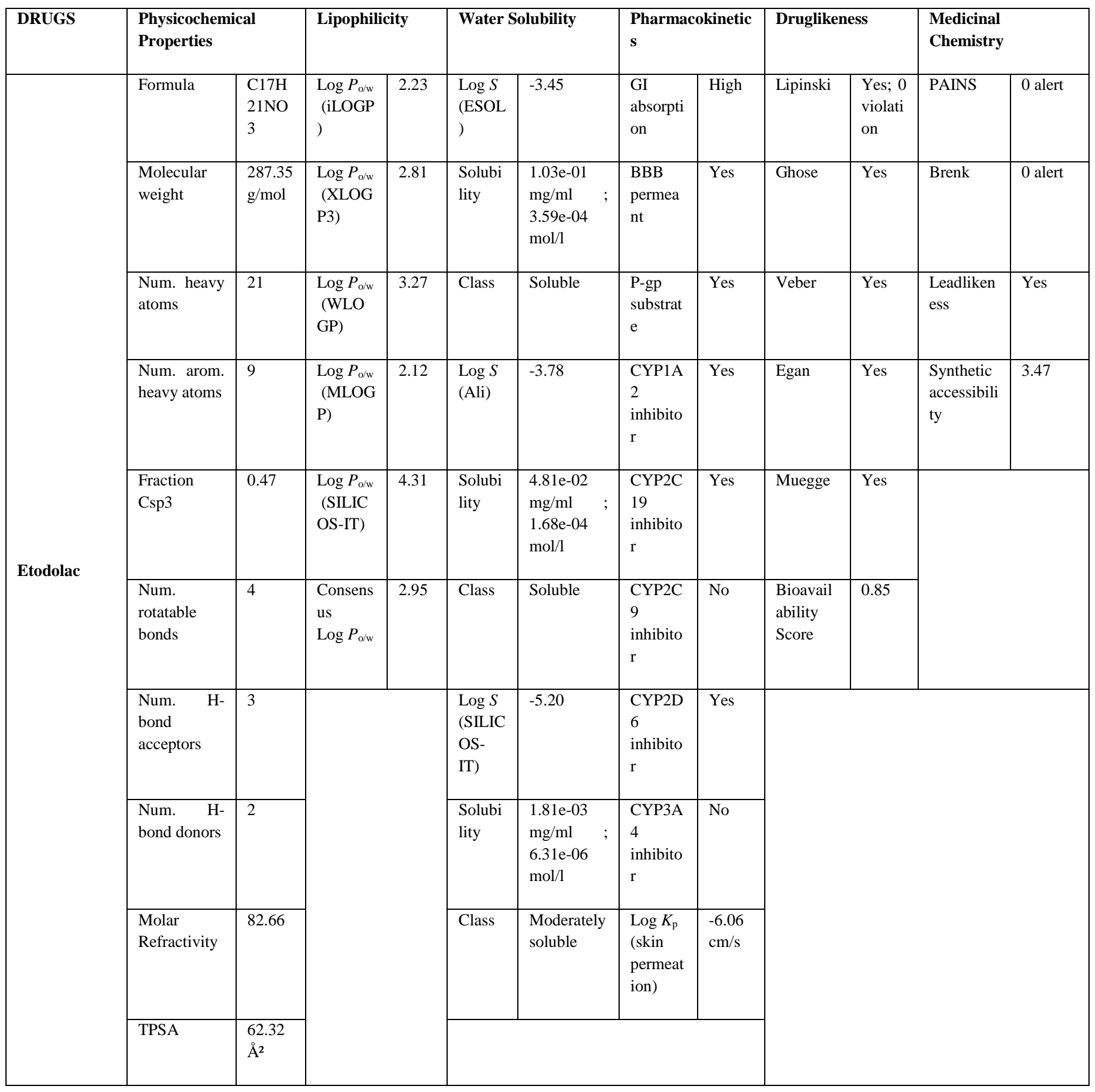

\section{Discussion}

A study examining the fluid from the lungs of 88 people with severe pneumonia caused by SARS-CoV2 infection concluded that once the virus reaches the lungs, it can infect 
macrophages, which respond by producing inflammatory molecules that attract T-cells. In turn, they produce a protein that stimulates macrophages to make more inflammatory molecules. This persistent lung inflammation could lead to some of the life-threatening consequences of SARSCoV2 infection [14]. Their study strongly supports the recommendation of using antiinflammatory drugs to prevent deaths from COVID-19. Another study concluded that the use of an anti-inflammatory or an immune modulator, to treat infectious diseases is profound. It's a really big deal. They found that patients receiving the combination treatment had a median recovery time of seven days, versus eight days for the remdesivir-only group. Also, patients receiving greater oxygen support or non-invasive ventilation had a recovery time of 10 days with the combination treatment, compared to 18 days with the control medication. They further stated that patients taking baricitinib and remdesivir had a $30 \%$ greater improvement in clinical status at day 15 compared to the control arm [15]. According to J. Jesus Naveja and his co-workers, the use of curcumin along with invermectin had many favorable effects against apoptosis, inflammation, pulmonary edema suppression, replication of RNA, and pathways associated with fibrosis in SARS-CoV 2 infection. Curcumin works as an anti-inflammatory agent by inhibiting cytokine storm, regulate inflammatory factors like COX-2 and interleukins that activate inflammatory responses [16]. An another study concluded that the risk of hospital administration of coronavirus patients with the rheumatic chronic inflammatory disease was significantly reduced by using NSAIDs [17].

We employed innovative access by gathering medicinal compounds that contain anti-viral and immunomodulatory activities to analyse their inhibitory interaction across proteins of virus. Within the viral protein, both structural proteins and non-structural proteins of the virus lies. For example, spike glycoprotein were the structural proteins that permit the viral compounds to attach themselves to the host cell ACE-2 receptor, (NSP-15, NSP-9) the non-structural proteins ease viral replication and along with these proteases inflect the manufacturing of diverse proteins via proteolytic cleavage $[18,19]$. In this study, our main objective was to achieve novel drug aspirants that comprise of three main characters one should retain their competent pharmacokinetic properties with the least toxicity, and to assure safety all along administration and must possess remarkable competency to interact with the targeted site of these viral proteins. 
MOD refiner \& PRODRG servers were used for the preparation of both proteins and ligands to remove any worse contacts that may include structural abnormality and undesirable potential energy that results in pseudo interaction. To drain out most feasible drug candidates developing hydrogen bonds except for Vander Waal interaction, with viral protein receptors active site residues and to execute basic screening of these ligands the subtle molecules were recycled and meanwhile combined to raccoon [20]. The compound that established hydrogen bonds with these fervid proteins were checked and further inspected for evaluating the interaction stability of RMSD ligand via ligand RMSD [21]. In between 0.9-1.5 A was the estimated figure of RMSD of these compounds which were regarded as satisfactory and balanced.

By GUSAR software [22] and SwissADME [23], the acute toxicity and pharmacokinetic attributes of these compounds were determined. To evoke the toxic response these compounds require a high dose because the toxicity figure of these compounds was comparatively depressive. The Class 4 chemicals that contained the bulk of compounds had characteristic benign pernicious effects (diarrhea and piloerection), whereas Class 5 chemicals compound possess few toxic effects [20]. Thus, the dosage of these compounds should be measured as means to escapade their whole advantages and avoid negative effects.

The main indication of this coronavirus disease was the onset of a cytokine storm that was prevented by these medicinal compounds moreover these compounds revitalize immunological responses. By the combination of these medicinal compounds with basic anti-viral medicines, collegially intensifying the inhibitory action, lowers the toxic effects [24] boosts tissue repair, and improves the sufferer's symptoms [25]. Assimilating these compounds with interim approved drugs may establish a fair immunological response against this infection. Along with this, these compounds also responsible for boosting up phagocytosis functions, and the proliferation of macrophages and neutrophils were also adjusted. By endorsing T-cells cytokine production they accelerate the activity of natural killer cells, adaptive immunity establishment, and stimulation of dendritic cells, which takes 4 to 7 days for activation. Thus through this intervention, the awful condition of infected individuals may be improved by boosting their immunity.

\section{Conclusion}


Different compounds that had an efficient pharmacokinetic profile, binding affinity, and low toxicity were passed through virtual screening in this study. These compounds became successfully filtered and were considered promising drug candidates for the treatment of SARS CoV2. The therapeutic efficacy of these compounds further utilized as lead compounds for clinical trials through in-vivo and in-vitro experiments. Furthermore, through this study mechanism of these drug compounds and their chemical affinity against SARS-CoV2 protein targets were also illuminated. So, the result of this study can be utilized as a vehicle obtained via computer simulation for clinicians and other medical researchers looking for promising antiSARS-CoV2 therapies.

\section{References}

1. Jiang F, Deng L, Zhang L, Cai Y, Cheung CW, Xia Z. Review of the Clinical Characteristics of Coronavirus Disease 2019 (COVID-19). J Gen Intern Med. 2020;35: 1545-1549. doi:10.1007/s11606-020-05762-w

2. Dashraath P, Wong JLJ, Lim MXK, Lim LM, Li S, Biswas A, et al. Coronavirus disease 2019 (COVID-19) pandemic and pregnancy. Am J Obstet Gynecol. 2020;222: 521-531. doi:https://doi.org/10.1016/j.ajog.2020.03.021

3. McCreary EK, Pogue JM. Coronavirus disease 2019 treatment: A review of early and emerging options. Open Forum Infectious Diseases. 2020. doi:10.1093/ofid/ofaa105

4. Hui DS, I Azhar E, Madani TA, Ntoumi F, Kock R, Dar O, et al. The continuing 2019nCoV epidemic threat of novel coronaviruses to global health \&\#x2014; The latest 2019 novel coronavirus outbreak in Wuhan, China. Int J Infect Dis. 2020;91: 264-266. doi:10.1016/j.ijid.2020.01.009

5. Singhal T. A Review of Coronavirus Disease-2019 (COVID-19). Indian J Pediatr. 2020;87: 281-286. doi:10.1007/s12098-020-03263-6

6. Gómez-Rial J, Rivero-Calle I, Salas A, Martinón-Torres F. Role of 
Monocytes/Macrophages in Covid-19 Pathogenesis: Implications for Therapy. Infect Drug Resist. 2020;13: 2485-2493. doi:10.2147/IDR.S258639

7. Giollo A, Adami G, Gatti D, Idolazzi L, Rossini M. Coronavirus disease 19 (Covid-19) and non-steroidal anti-inflammatory drugs (NSAID). Ann Rheum Dis. 2021;80: e12--e12. doi:10.1136/annrheumdis-2020-217598

8. Ojha PK, Kar S, Krishna JG, Roy K, Leszczynski J. Therapeutics for COVID-19: from computation to practices - where we are, where we are heading to. Molecular Diversity. Springer International Publishing; 2020. doi:10.1007/s11030-020-10134-X

9. Cusinato J, Cau Y, Calvani AM, Mori M. Repurposing drugs for the management of COVID-19. Expert Opin Ther Pat. 2020; 1-13. doi:10.1080/13543776.2021.1861248

10. Mishra D, Mishra A, Chaturvedi VK, Singh MP. An overview of COVID-19 with an emphasis on computational approach for its preventive intervention. 3 Biotech. 2020;10: 435. doi:10.1007/s13205-020-02425-9

11. Marinho EM, Batista de Andrade Neto J, Silva J, Rocha da Silva C, Cavalcanti BC, Marinho ES, et al. Virtual screening based on molecular docking of possible inhibitors of Covid-19 main protease. Microb Pathog. 2020;148: 104365. doi:10.1016/j.micpath.2020.104365

12. Zhou Y, Hou Y, Shen J, Huang Y, Martin W, Cheng F. Network-based drug repurposing for novel coronavirus 2019-nCoV/SARS-CoV-2. Cell Discov. 2020;6: 14. doi:10.1038/s41421-020-0153-3

13. Singh P, Mishra N, Singh N, Nisha R, Pal RR, Singh S, et al. Credible Protein Targets and Curative Strategies for COVID-19: a Review. SN Compr Clin Med. 2020;2: 2067-2076. doi:10.1007/s42399-020-00526-3

14. Grant RA, Morales-Nebreda L, Markov NS, Swaminathan S, Querrey M, Guzman ER, et al. Circuits between infected macrophages and $\mathrm{T}$ cells in SARS-CoV-2 pneumonia. Nature. 2021; doi:10.1038/s41586-020-03148-w

15. Titanji BK, Farley MM, Mehta A, Connor-Schuler R, Moanna A, Cribbs SK, et al. Use of 
baricitinib in patients with moderate and severe COVID-19. Clin Infect Dis. 2020;

16. Russell B, Moss C, Rigg A, Van Hemelrijck M. COVID-19 and treatment with NSAIDs and corticosteroids: Should we be limiting their use in the clinical setting? Ecancermedicalscience. 2020;14: 1023. doi:10.3332/ecancer.2020.1023

17. Freites Nuñez DD, Leon L, Mucientes A, Rodriguez-Rodriguez L, Font Urgelles J, Madrid García A, et al. Risk factors for hospital admissions related to COVID-19 in patients with autoimmune inflammatory rheumatic diseases. Ann Rheum Dis. 2020;79: 1393 LP - 1399. doi:10.1136/annrheumdis-2020-217984

18. Kipshidze N, Iversen P, Porter TR, Kipshidze N, Siddiqui F, Dangas G, et al. Targeted, Site-Specific, Delivery Vehicles of Therapeutics for COVID-19 Patients. Brief Review. Clin Appl Thromb. 2020;26: 1076029620954911.

19. Hoffmann M, Kleine-Weber H, Schroeder S, Krüger N, Herrler T, Erichsen S, et al. SARS-CoV-2 Cell Entry Depends on ACE2 and TMPRSS2 and Is Blocked by a Clinically Proven Protease Inhibitor. Cell. 2020;181: 271-280.e8. doi:10.1016/j.cell.2020.02.052

20. Salman S, Shah FH, Idrees J, Idrees F, Velagala S, Ali J, et al. Virtual screening of immunomodulatory medicinal compounds as promising anti-SARS-CoV-2 inhibitors. Future Virol. 2020;15: 267-275. doi:10.2217/fvl-2020-0079

21. Mittal L, Kumari A, Srivastava M, Singh M, Asthana S. Identification of potential molecules against COVID-19 main protease through structure-guided virtual screening approach. J Biomol Struct Dyn. 2020; 1-19. doi:10.1080/07391102.2020.1768151

22. Mothay D, Ramesh K V. Binding site analysis of potential protease inhibitors of COVID19 using AutoDock. VirusDisease. 2020;31: 194-199. doi:10.1007/s13337-020-00585-z

23. Tian S, Hu N, Lou J, Chen K, Kang X, Xiang Z, et al. Characteristics of COVID-19 infection in Beijing. J Infect. 2020;80: 401-406. doi:https://doi.org/10.1016/j.jinf.2020.02.018

24. Kandeel M, Al-Nazawi M. Virtual screening and repurposing of FDA approved drugs 
against COVID-19 main protease. Life Sci. 2020;251: 117627. doi:https://doi.org/10.1016/j.lfs.2020.117627

25. Jin Z, Du X, Xu Y, Deng Y, Liu M, Zhao Y, et al. Structure-based drug design, virtual screening and high-throughput screening rapidly identify antiviral leads targeting COVID19. bioRxiv. 2020; 2020.02.26.964882. doi:10.1101/2020.02.26.964882 